\title{
Electrochemical sensing of biomarker for diagnostics of bacteria-specific infections
}

\begin{abstract}
Aim: Pseudomonas aeruginosa is a pathogen that is prevalent in serious infections in compromised patients worldwide. A unique virulence factor of this bacterium is the redox-active molecule pyocyanin, which is a potential biomarker for the identification of $P$. aeruginosa infections. Here we report a direct, selective and rapid detection technique of pyocyanin. Materials \& methods: Pyocyanin was detected by amperometry at a relatively high potential where the pyocyanin signal was unaffected by background contributions. Results \& conclusion: Pyocyanin was detected at concentrations down to $125 \mathrm{nM}$ in a $50 \mu \mathrm{M}$ mixture of interfering compounds with a reproducibility of $r^{2}=0.999(n=5)$ within $200 \mathrm{~s}$. The results document a step toward a point-of-care technique for diagnosis of $P$. aeruginosa infections.
\end{abstract}

First draft submitted: 12 April 2016; Accepted for publication: 23 June 2016; Published online: 27 July 2016

Keywords: amperometry $\bullet$ bacteria $\bullet$ biosensors $\bullet$ cystic fibrosis $\bullet$ detection $\bullet$ diagnosis - electrochemistry • electrodes $\bullet$ Pseudomonas aeruginosa $\bullet$ pyocyanin

Pseudomonas aeruginosa is an opportunistic pathogen that rarely infects healthy individuals but is life-threatening in critically ill patients. It is one of the most common causes of infections in burn injuries, airways, urinary tract, wounds and blood infections. Furthermore, it is a leading cause of hospitalacquired infections, having a high mortality rate especially among patients with compromised immune systems such as premature infants and cancer patients [1-3]. Thus, the Infectious Diseases Society of America has listed $P$. aeruginosa as one of the six bacteria that are responsible for the majority of healthcare-associated infections [4].

$P$. aeruginosa infections are difficult to treat due to the increasing resistance and tolerance to antibiotics $[5,6]$. The increasing resistance of bacteria is partly due to the late diagnosis and the overprescription of antibiotics to patients that show symptoms of infection [7]. Early and fast detection of this serious opportunistic pathogen could lead to more targeted antibiotic prescription, which will not only lead to a more efficient treatment of the patient but also reduce the emergence of antibiotic resistance [8,9].

The identification of $P$. aeruginosa is currently conducted by bacterial culturing on agar plates with selective growth compounds that favor the growth of the target bacterial strain. However, this process takes several days and requires a relatively high initial bacterial strain concentration in order to determine growth. Another approach is RNA sequencing to identify the presence of the suspected bacteria. Likewise, this method is expensive and time consuming.

$P$. aeruginosa secretes virulence factors that promote infections in host tissue. One of these virulence factors is pyocyanin that initiates inflammation and leads to apoptosis of neutrophils in infected patients $[10,11]$. Pyocyanin is exclusively secreted by $P$. aeruginosa, giving this molecule a great potential to be used as a diagnostic biomarker. Thus, high-
Fatima AlZahra'a

Alatraktchi*,1,2,3, Helle Krogh Johansen ${ }^{3,4}$, Søren Molin ${ }^{2,3}$ \&

Winnie Edith Svendsen ${ }^{1}$

'Department of Micro- \&

Nanotechnology, Technical University of Denmark, Kgs-Lyngby, Denmark

${ }^{2}$ Department of Biotechnology \& Biomedicine, Technical University of Denmark, Kgs-Lyngby, Denmark ${ }^{3}$ Novo Nordisk Foundation Center for Biosustainability, Technical University of Denmark, Hørsholm, Denmark ${ }^{4}$ Cystisk Fibrose Klinikken \& Klinisk Mikrobiologisk Afdeling - Rigshospitalet, Copenhagen, Denmark

*Author for correspondence: faaat@nanotech.dtu.dk 
resolution monitoring of pyocyanin would assist in the early diagnosis and treatment of these life-threatening infections [12].

The most common approach for the detection of pyocyanin is spectrophotometry as pyocyanin has a characteristic bluish color. However, this technique is time consuming due to the necessity of sample pretreatment to avoid background noise. A more modern approach is the use of electrochemistry to detect pyocyanin. Electrochemical measurements allow point-ofcare diagnosis without any pretreatments or lengthy traditional measurement procedures [13-15].

Electrochemical detection of pyocyanin has been reported in research; however, it has always been performed by voltammetric methods, as cyclic voltammetry, square wave voltammetry and differential pulse voltammetry [16-19]. In contrary, amperometric methods have so far not been used to quantify pyocyanin. This is most likely due to the difficulty of distinguishing electrochemical contributions from other redoxactive compounds that exists in human samples. Nevertheless, the same issue regarding interference with the signals of interest is still present in voltammetric techniques. Most of the studies use characteristic potential peaks of pyocyanin of approximately $-200 \mathrm{mV}$, claiming that no other interferents are electrochemically active at this potential. However, it is unclear as to what extent this peak would interfere with background signals from patient samples, like blood, urine or sputum. In a former study we demonstrated that human saliva and artificial sputum have cyclic voltammetric peaks overlapping with the commonly used peaks of pyocyanin [13]. This limits the diagnostic possibilities in samples from, for example, lung infected patients.

Due to the chemical nature of pyocyanin, it is involved in multiple redox-based interactions. Pyocyanin is a membrane-permeable molecule that enters cells and reacts with intracellular metabolites like nicotinamide adenine dinucleotide phosphate (NADPH) and nicotinamide adenine dinucleotide (NADH) [20]. If any of these metabolites are released to the extracellular environment, it is likely that their signal would interfere with the electrochemical footprint of pyocyanin. There exist around $20 \times 10^{9}$ bacterial cells orally in approximately $0.2 \mathrm{ml}$ saliva [21]. Assuming that all cells were lysed it would correspond to around $0.2 \mathrm{nM}$ $\mathrm{NADH}$. As we aim to detect pyocyanin in nanomolar ranges, this concentration is comparable to the target signal. Thus, it is highly important to take into account this type of metabolites and redox-active compounds when interpreting the electrochemical profile of pyocyanin to achieve selective detection.

Here we describe a method of amperometric selective identification and quantification of pyocyanin among interfering compounds. In amperometric measurements, the current is integrated over relatively long time intervals, giving a much better $S / N$ in comparison to voltammetric techniques. The enhanced $\mathrm{S} / \mathrm{N}$ should result in lower detection limits than those obtained by voltammetric quantification. A procedure is provided for how to identify and quantify pyocyanin among other redox-active chemicals by amperometric measurements down to nanomolar ranges. Finally, this method is compared with spectrophotometric measurements of pyocyanin.

\section{Materials \& methods}

\section{Reagents}

Stock solutions of $100 \mu \mathrm{M}$ pyocyanin (P004625MG, SIGMA, Copenhagen, Denmark), pyoverdine (P8374-1MG, SIGMA, Copenhagen, Denmark), nicotinamide adenine dinucleotide phosphate hydrate (NADP) (N5755 SIGMA, Copenhagen, Denmark), NADPH (N5130 SIGMA, Copenhagen, Denmark), nicotinamide adenine dinucleotide hydrate (NAD) (N1636, SIGMA-ALDRICH, Copenhagen, Denmark), NADH (N4505, SIGMA, Copenhagen, Denmark), phenazine- $\mathrm{C}_{12} \mathrm{H}_{8} \mathrm{~N}_{2}$ (P13207 ALDRICH, Copenhagen, Denmark) and Lysogeny broth medium (LB) were prepared in MilliQ water, respectively.

\section{Preparation of samples}

Pyocyanin, pyoverdine, NAD, NADH, NADP, NADPH and LB were prepared in $100 \mu \mathrm{M}$ concentrations in MilliQ water, respectively. From this several dilution series were arranged. A chemical mixture (CM) of all the compounds without pyocyanin was prepared and a dilution series from 0 to $100 \mu \mathrm{M}$ was made. Pyocyanin dilutions from $125 \mathrm{nM}$ to $90 \mu \mathrm{M}$ were prepared in CM backgrounds of $0,5,25$ and $50 \mu \mathrm{M}$, respectively. The artificial sputum was prepared according to [22].

\section{Electrochemical profiling \& quantification protocol}

All electrochemical experiments were performed using disposable screen-printed electrodes with a threeelectrode configuration featuring gold working and counter electrode (CEs) and a silver reference electrode (C223AT, Dropsens, Spain). The measurements were recorded by a potentiostat (Metrohm Autolab, The Netherlands). All measurements were conducted by placing a drop of $70.8 \mu \mathrm{l}$ on fresh electrodes for each experiment. A schematic representation of the screenprinted sensor with a sample drop and the electric circuit of the three-electrode configuration are shown in Figure $1 \mathrm{~A}$ and $\mathrm{B}$. The electrode, where the electrochemical reaction takes place, is the working electrode 
(WE). The CE closes the circuit, while the reference electrode measures the voltage between the electrolyte and WE.

The individual chemicals were characterized by cyclic voltammetry with a potential of $-1.0-1.0 \mathrm{~V}$ and a scan rate of $0.05 \mathrm{~V} / \mathrm{s}$, as previously described by Alatraktchi et al. [13].

Amperometric measurements were obtained by recording the decay in the measured signal from the electrochemical interaction between the sample and the electrodes. Five replicates were taken for each sample on a fresh sensor. Amperometric measurements of
MilliQ Water, CM $50 \mu \mathrm{M}$, pyocyanin $50 \mu \mathrm{M}$ and 50 $\mu \mathrm{M}$ pyocyanin in $50 \mu \mathrm{M} \mathrm{CM}$ were recorded at steps between 0.10 and $0.90 \mathrm{~V}$. CM concentration between 10 and $100 \mu \mathrm{M}$ was measured at 0.82 and $0.45 \mathrm{~V}$, respectively. The same was conducted for pyocyanin in 5,25 and $50 \mu \mathrm{M} \mathrm{CM}$ backgrounds. All the measurements were conducted versus the Ag reference electrode.

Data were handled in Autolab NOVA 1.10. The amperometric signals were integrated between 0 and $200 \mathrm{~s}$ to obtain the accumulated charge for each amperometric measurement.

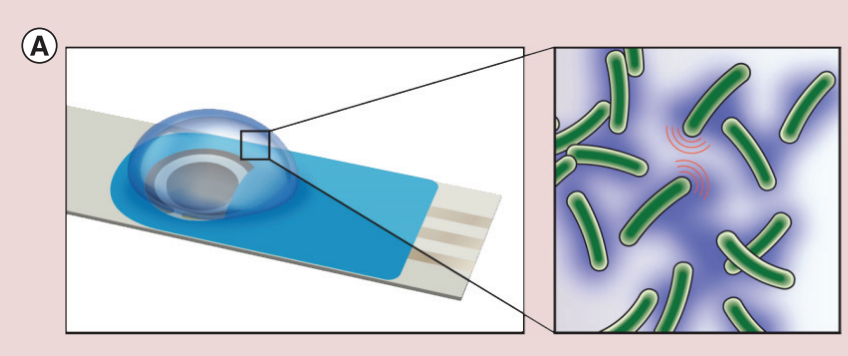

(B)

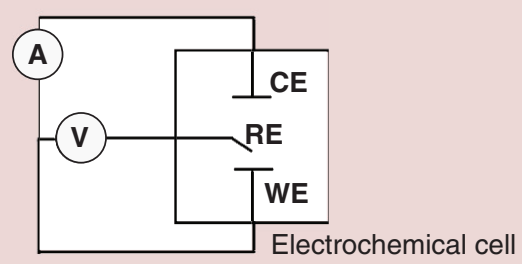

(C)

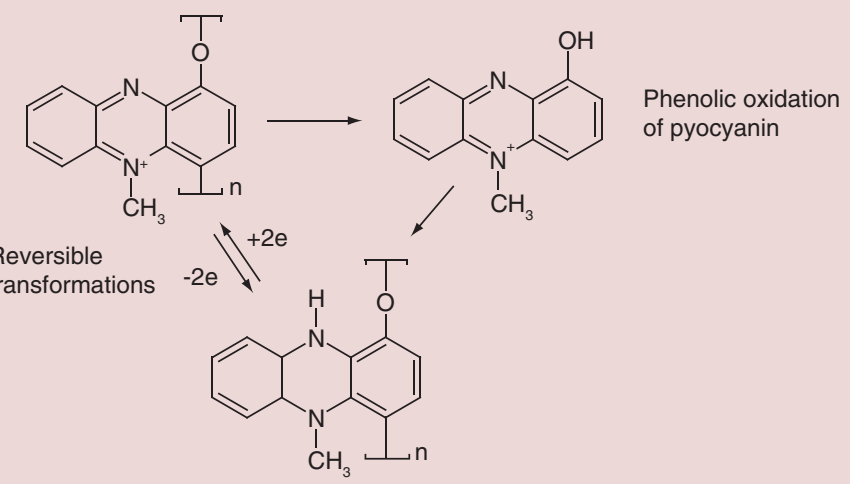

(D)

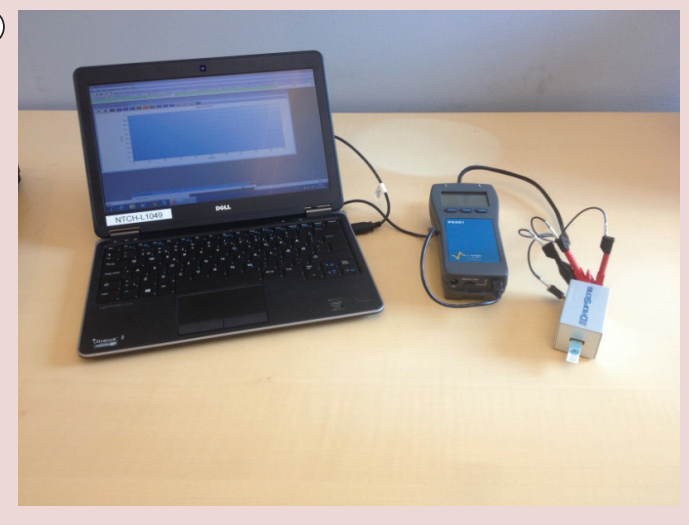

Figure 1. Concept of electrochemical detection of pyocyanin production. (A) Schematic of the sensing chip with a sample of bacteria that produce pyocyanin. (B) Equivalent circuit for the three-electrode configuration setup consisting of a working electrode, counter electrode and reference electrode in an electrochemical cell and (C) the reactions pyocyanin undergoes when potential is applied to the system. (D) The experimental setup where the sensor is connected to the potentiostat that reads and saves the current values on a computer.

CE: Counter electrode; RE: Reference electrode; WE: Working electrode.

(A) Reprinted with permission from [13]. 


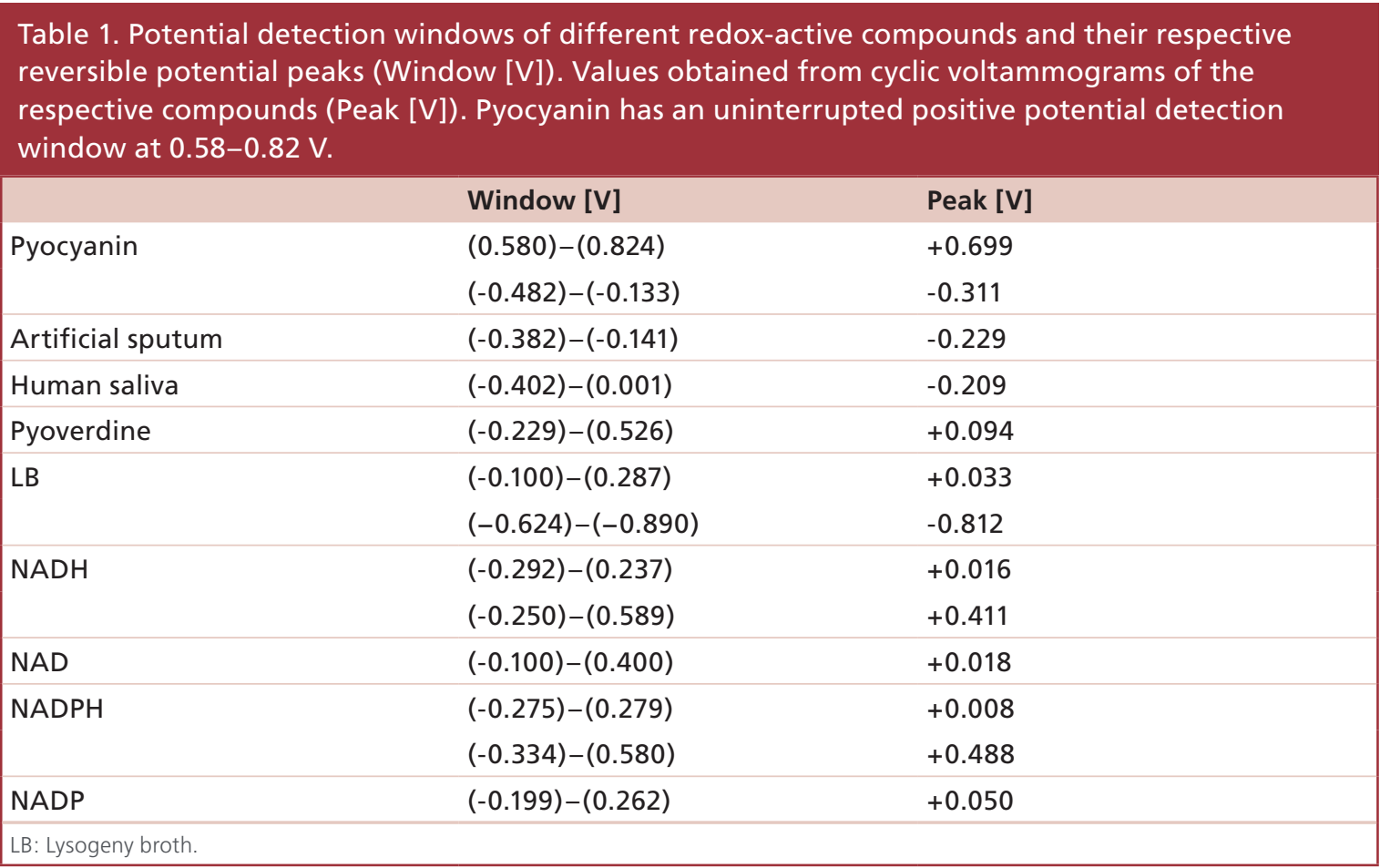

\section{Spectrophotometry}

The detection limit of spectrophotometry was examined by preparing pyocyanin calibration standards in the range of $0-100 \mu \mathrm{M}$ and measuring using an UVspectrophotometer (Shimadzu, UV-1800). The wavelength of $695 \mathrm{~nm}$ was used to obtain pyocyanin reading with MilliQ water as the reference. The extinction coefficient of pyocyanin is 5.816 .

\section{Detection in bacterial culture}

PAO1 bacteria were cultured in artificial sputum and left for overnight growth. The accumulated pyocyanin content in the bacterial culture was measured by amperometry at $0.82 \mathrm{~V}$ versus the Ag reference electrode by pipetting a sample on a fresh sensor. Then a new sample was diluted 1:2 in artificial sputum medium and measured by amperometry at $0.82 \mathrm{~V}$ versus the $\mathrm{Ag}$ reference electrode for $200 \mathrm{~s}$. Artificial sputum without bacteria was similarly measured by amperometry to account for the background signal. This measurement was conducted using a portable potentiostat (PG851, BioLogic, France). The experimental setup is seen in Figure 1D.

\section{Results}

Choosing optimal potential for amperometric measurements

Voltammetric profiling of the redox-active metabolites was conducted to characterize the most optimal detection potential of pyocyanin to be used as input poten- tial in the amperometric experiments. The potential at which the current peak occurs is dependent on the redox potential of the measured species. The potential detection window of a compound was determined by the start base and the end base of a peak event. The potential detection windows of the characterized compounds are listed in Table 1. Pyocyanin undergoes chemical transformations during the potential sweep as seen in Figure 1C. Pyocyanin undergoes reversible transformations at negative potentials between -0.482 and $-0.133 \mathrm{~V}$ and nonreversible phenolic oxidation at the positive potentials between 0.580 and $0.824 \mathrm{~V}$. It is seen that the potential window of $0.58-0.82 \mathrm{~V}$ of pyocyanin is higher than the windows of the other compounds and lies exclusively outside any overlap.

Choosing a relatively high potential as $0.699 \mathrm{~V}$ for the amperometric measurements gives rise to difficulties due to loss of specificity. The electric current upstream from the lower potential compounds can flow to the detection potential of $0.699 \mathrm{~V}$. This is a challenge in attaining high sensitivity. However, this can be corrected by choosing the appropriate potential for pyocyanin quantification with regard to the $\mathrm{CM}$ background. Thus, characterizing the mixture of interfering compounds is as important as choosing the appropriate applied potential. CM, pyocyanin and pyocyanin + CM was, therefore, characterized by amperometric means. Figure $1 \mathrm{~A}$ shows a typical amperometric measurement of $50 \mu \mathrm{M}$ pyocyanin + $50 \mu \mathrm{M}$ CM detected at $0.82 \mathrm{~V}$. It is seen how the 
current drops over time as described by the Cottrell equation. The integration of the area beneath the curve is a measure of the accumulated charge obtained from the sample in the time interval of the integral. The replicates of the same experiment reveal nearly perfect accuracy with an $r^{2}=0.999$ and a similar accuracy for measurements conducted at lower applied potential of $0.45 \mathrm{~V}$ (Figure 2B). The accuracy of the replicates comes from integrating the current over a relatively long time interval which minimizes the noise coming from fluctuations in the current decay.

MilliQ water, CM, pyocyanin and pyocyanin + $\mathrm{CM}$ were measured by amperometry at step potentials from 0.10 to $0.90 \mathrm{~V}$ (Figure 3A). The MilliQ water has the lowest accumulated charge value at all the applied potentials. Next comes CM that similarly to MilliQ water has an almost exponential increase as the potential is increased. On the other hand, pyocyanin is close to background values that has a relatively humble increase at $0.50 \mathrm{~V}$ and begins to express significantly high accumulated charge at $0.60 \mathrm{~V}$. This is consistent with the potential windows shown in Table 1, which states that pyocyanin is detectable starting from $0.58 \mathrm{~V}$. The situation is similar with pyocyanin + CM, however, here the increase in accumulated charge begins at $0.45 \mathrm{~V}$ which partly can be assigned to the $\mathrm{CM}$ contribution. At this potential the pyocyanin + CM is higher than the pyocyanin measurement. Table 1 shows that $0.45 \mathrm{~V}$ is not within any of the potential windows of pyocyanin which also indicates that the detected increase in signal at $0.45 \mathrm{~V}$ is due to the $\mathrm{CM}$ content in the sample.

Comparing the accumulated charge of the pyocyanin measurements to the pyocyanin + CM measurements it is observed that the latter is reduced compared with pyocyanin. A damping of the pyocyanin signal is thus appearing when a CM background is present in the sample. The pyocyanin signal is probably damped due to the presence of NADH and NADPH in the samples. In real conditions, dead cells would release metabolites like NAD, NADH, NADP and NADPH to the extracellular environment. In addition, pyocyanin is a membrane-permeable molecule that enters cells and accepts electrons from NADH and NADPH. This is all factors that would influence the signal of pyocyanin.

Subtracting the accumulated charge from pyocyanin + CM from the charge of pyocyanin at each applied potential, it is seen that the damping is close to zero coulomb in the range from 0.10 to $40 \mathrm{~V}$ (Figure 3B). The damping is less than zero coulomb at $0.45 \mathrm{~V}$, meaning that the pyocyanin + CM level was higher than the level of pyocyanin at this potential.
Subsequently, the damping is significantly increasing in the range of $0.50-0.90 \mathrm{~V}$ with the lowest damping occurring at $0.82 \mathrm{~V}$. As this potential is also the end base of the pyocyanin detection window obtained in Table 1, this was chosen as the applied potential in the further pyocyanin quantification experiments. Additionally, the potential of $0.45 \mathrm{~V}$ was also chosen to characterize the trend in CM contribution in the further analyses.

\section{Characterizing the contribution from background compounds}

A dilution series of $\mathrm{CM}$ was measured by amperometry with applied potentials of 0.45 and $0.82 \mathrm{~V}$, respectively (Figure 4). At $0.82 \mathrm{~V}$, the $\mathrm{CM}$ starts gaining signal above MilliQ water when it exceeds $20 \mu \mathrm{M}$. Then, the accumulated charge increases linearly with concentration up to $80 \mu \mathrm{M}$ where it reaches a saturated level. As for the measurements at $0.45 \mathrm{~V}$ a slight increase

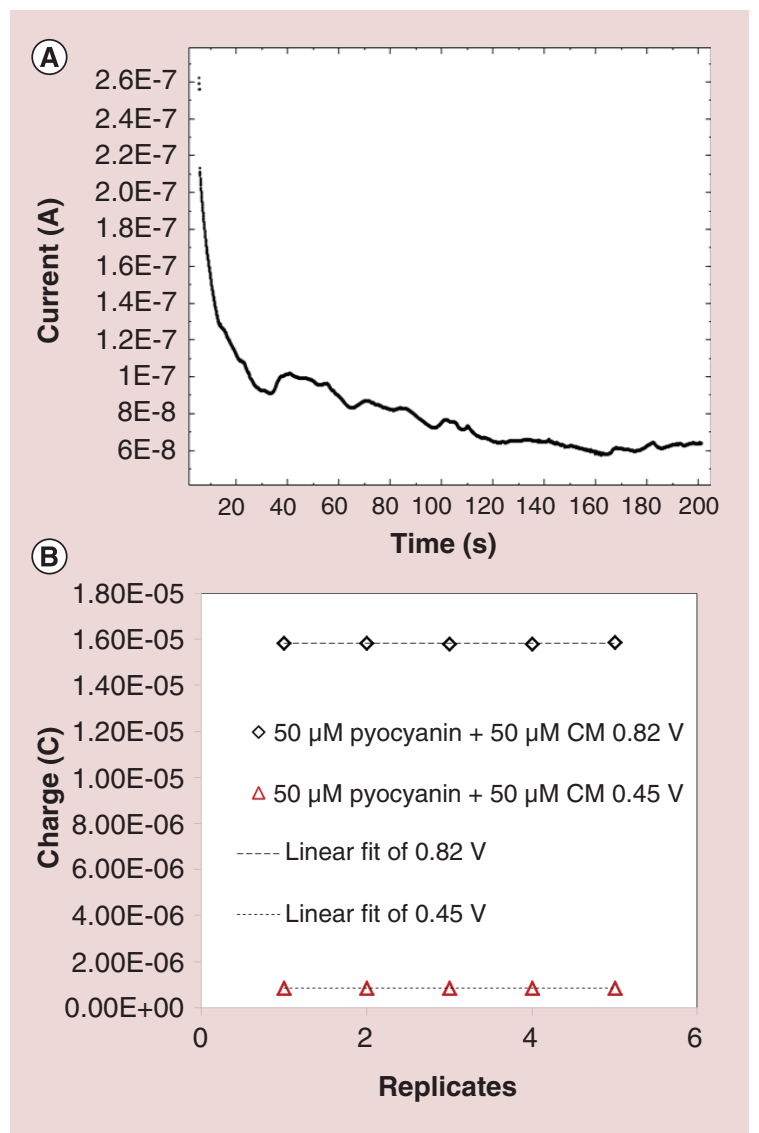

Figure 2. Amperometric measurements of $50 \mu \mathrm{M}$ pyocyanin + $50 \mu \mathrm{M}$ chemical mixture (CM). (A) Typical amperometric measurement showing the drop in current as function of time. (B) Replicates of the amperometric measurments after integrating the curves from 0 to $200 \mathrm{~s}$. The accumulated charge of a measurement is highly reproducible with an $r^{2}=0.999$ $(n=5)$. 


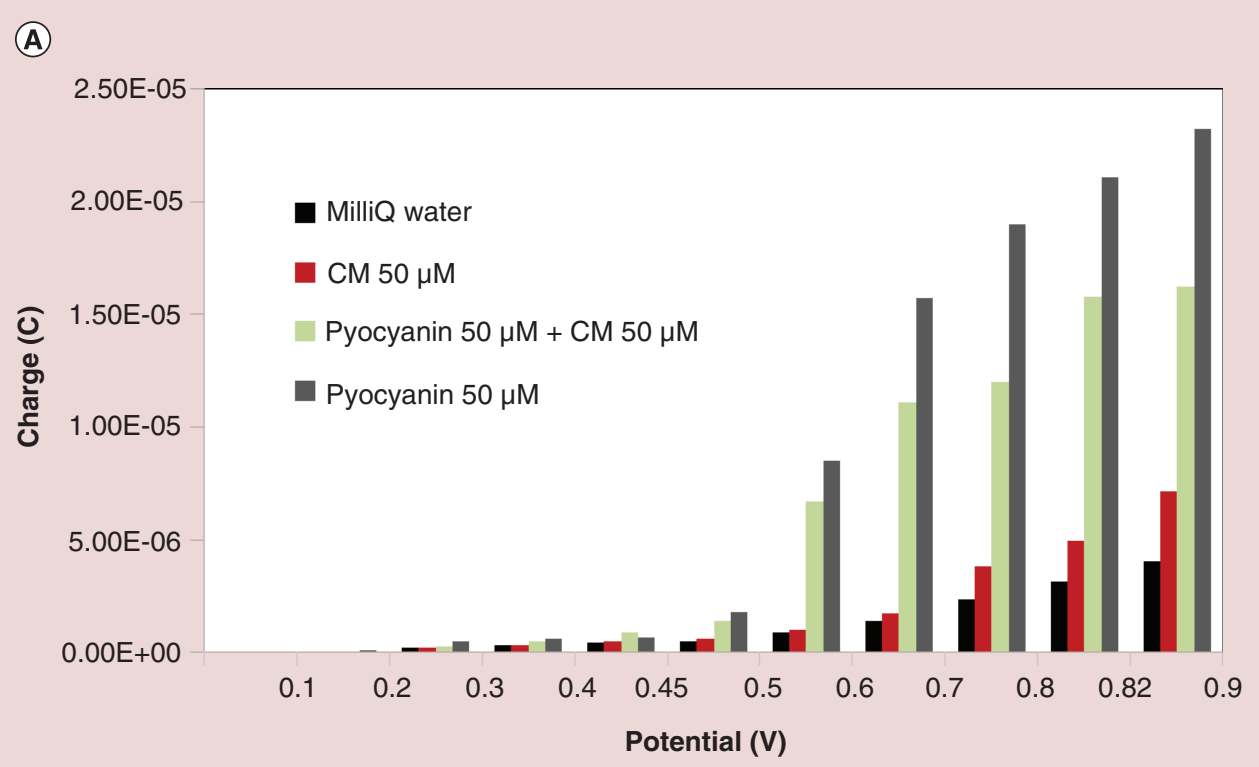

(B)

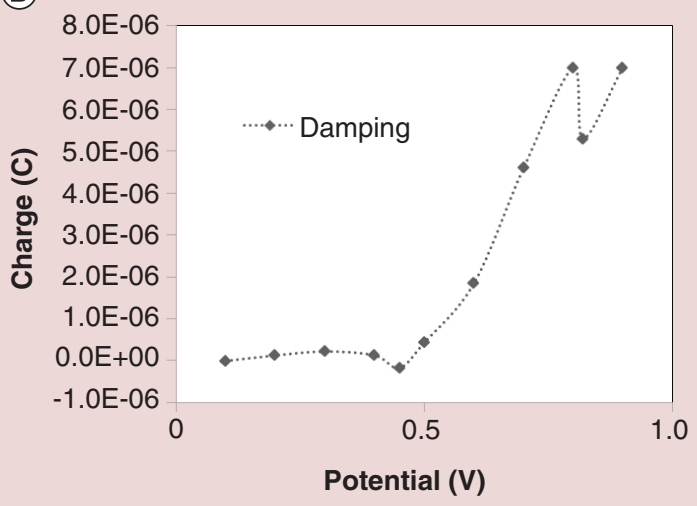

Figure 3. Accumulated charge as function of applied potential to amperometric measurements. (A) Accumulated charge obtained from amperometric measurements of MilliQ water, $50 \mu \mathrm{M}$ chemical mixture (CM) and $50 \mu \mathrm{M}$ pyocyanin $+50 \mu \mathrm{M} \mathrm{CM}$ at different potentials ranging from $0.10 \mathrm{~V}$ to $0.90 \mathrm{~V}$. Pyocyanin starts to show significant increase in accumulated charge at $0.50 \mathrm{~V}$. The pyocyanin signals are damped when detected in a mixture of interfering compounds compared with detecting it in its pure form. (B) The damping of accumulated charge as function of potential. In the pyocyanin detectable range the lowest damping is achieved at $0.82 \mathrm{~V}$ (arrow).

in accumulated charge is observed from $4.18 \times 10^{-7} \mathrm{C}$ to $5.48 \times 10^{-7} \mathrm{C}$. The saturation is believed to be due to the fact that the measurements are conducted at a potential that is only specific for pyocyanin. Therefore, the pyocyanin signal continues to rise proportionally with the concentration, whereas the background is not affected by the high but yet nonspecific potential for the compounds in CM.

From Figure $3 \mathrm{~A}$ it is seen that pyocyanin in a $\mathrm{CM}$ gives rise to significantly higher accumulated charge than $\mathrm{CM}$ alone. Thus, it is assumed that the saturation level obtained in Figure 4 is the maximum limit of background contribution and, therefore, constitutes the lower baseline of detectable pyocyanin that does not interfere with background signals.

\section{Selective pyocyanin quantification using amperometry}

The pyocyanin dilutions detected at $0.82 \mathrm{~V}$ in $\mathrm{CM}$ concentrations of 5, 25 and $50 \mu \mathrm{M}$ showed overlapping amperometric measurements for each pyocyanin concentration. The respective accumulated charge values were identical, regardless of the concentration of the CM background they were in. The proportionality of the amperometric measurements with pyocyanin concentration is observed in Figure 5. Figure 5A shows the pyocyanin range from 1 to $90 \mu \mathrm{M}$ while Figure $5 B$ shows the current measurements for the submicromolar range. The lowest detected concentration was $125 \mathrm{nM}$. The inset shows the linearity of the corresponding accumulated charge of the investigated concentration range having an $\mathrm{r}^{2}$ of 0.998 . 
The typical amperometric measurements of the same pyocyanin + CM dilutions at $0.45 \mathrm{~V}$ are seen in Figure 6A. The increase of signal is proportional with the concentration of pyocyanin, yet the increase in current is relatively humble. The amperometric measurements were translated to accumulated charge as seen in Figure 6B. Although the pyocyanin + CM measurements at $0.82 \mathrm{~V}$ resulted in identical accumulated charges of the different concentrations despite the $\mathrm{CM}$ background, a remarkable difference in accumulated charge was observed at $0.45 \mathrm{~V}$ when the $\mathrm{CM}$ concentration differed. The pyocyanin concentration in 50 $\mu \mathrm{M}$ background increased up to $40 \mu \mathrm{M}$, hereafter the increase was damped. When the CM background was lowered to $25 \mu \mathrm{M}$, the slope of the increase in accumulated charge was lowered at $25 \mu \mathrm{M}$. For pyocyanin measurements in $5 \mu \mathrm{M} \mathrm{CM}$, the shift in slope started at $5 \mu \mathrm{M}$ pyocyanin, obtaining steady state after $20 \mu \mathrm{M}$. Common for these measurements is that the slope of the accumulated charge decreases when the pyocyanin concentration becomes higher than the concentration of the CM. This observation is consistent with the results obtained in Figure 2 that shows signal damping when $\mathrm{CM}$ and pyocyanin are combined in one sample. It is also worth noticing that the $\mathrm{CM}$ accumulated charge approximates the value of $50 \mu \mathrm{M} \mathrm{CM}$ as the CM concentration decreases. Thus, the amperometric measurements at $0.45 \mathrm{~V}$ assist in understanding the background of the sample that pyocyanin is detected in.

The lowest detected pyocyanin concentration of $125 \mathrm{nM}$ has a corresponding accumulated charge of $4.80 \times 10^{-7} \mathrm{C}$ at $0.45 \mathrm{~V}$. The $50 \mu \mathrm{M} \mathrm{CM}$ contribution at this potential was $4.75 \times 10^{-7} \mathrm{C}$. Therefore, the $125 \mathrm{nM}$ is considered the LOD.

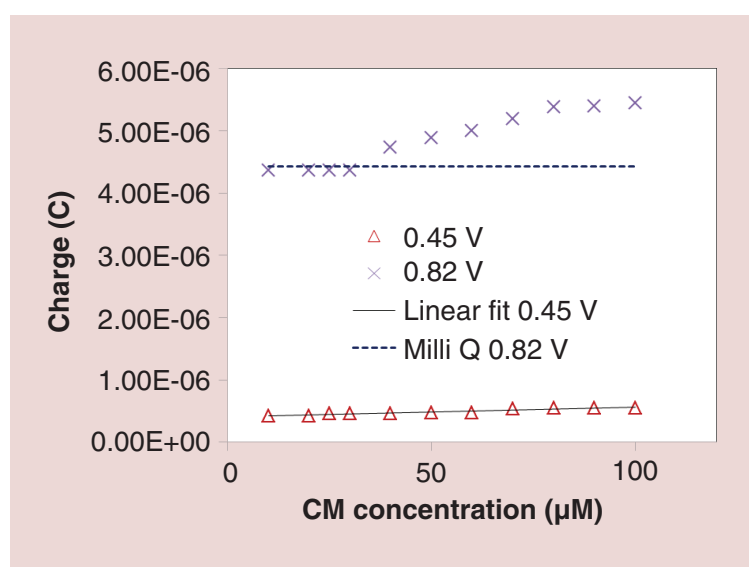

Figure 4. The charge accumulations of increasing chemical mixture (CM) concentration obtained from amperometric measurements. The accumulated charge of $\mathrm{CM}$ concentrations measured at $0.82 \mathrm{~V}$ shows increasing behavior that is saturated at high CM levels. When measuring at applied potential of $0.45 \mathrm{~V}$, the charge accumulation shows a slight increase.

\section{Detection limit of pyocyanin among}

\section{interferents by spectrophotometry}

The detection limit of pyocyanin in MilliQ water, 50 and $100 \mu \mathrm{M} \mathrm{CM}$, respectively, was investigated by spectrophotometry (Figure 7). Linear relation between absorbance and pyocyanin concentration was found, where the addition of CM shifted the absorbance level. The measurements below the background levels were nonlinear (data not shown). The lower detection limit of pyocyanin using spectrophotometry is also dependent on the concentration of CM in the samples. The lowest detected concentration was $1 \mu \mathrm{M}$ pyocyanin in water. The detection limit was $2.5 \mu \mathrm{M}$ when detecting in a CM background of $50 \mu \mathrm{M}$. These values are
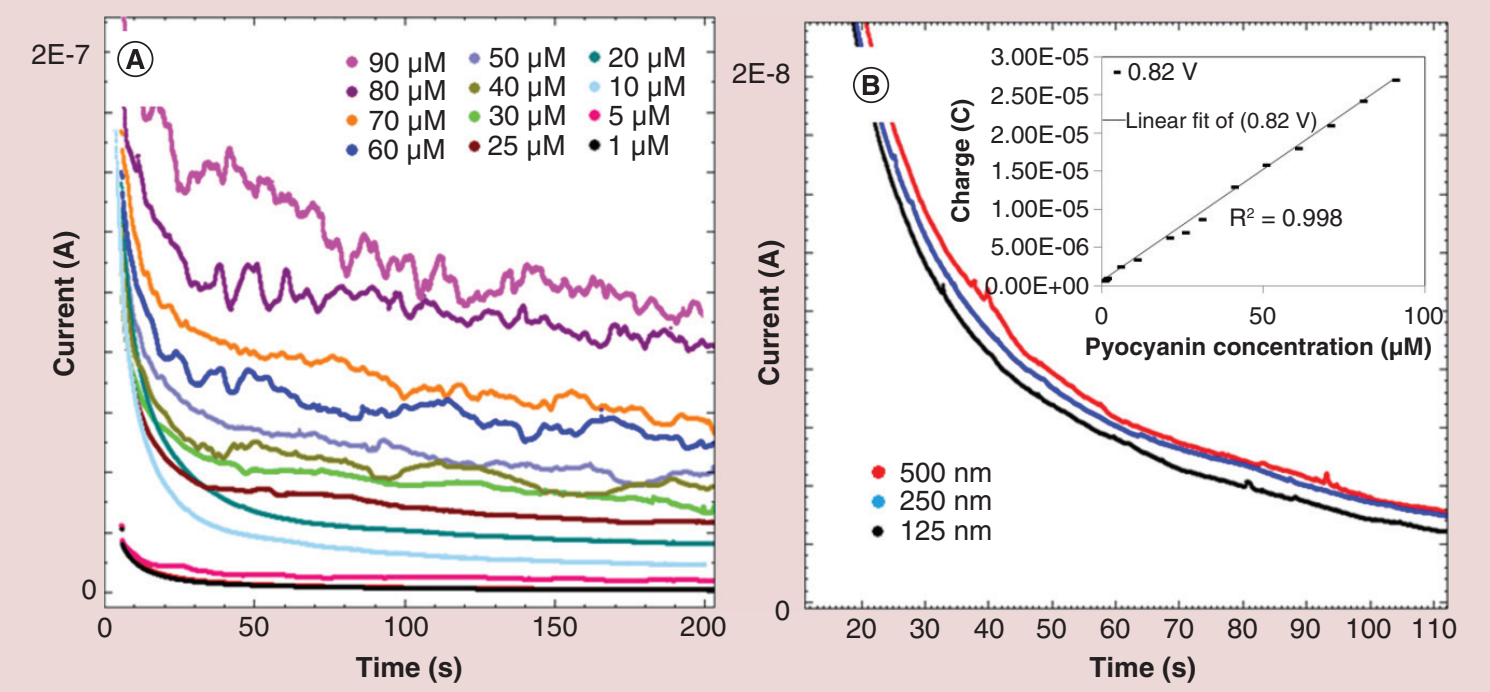

Figure 5. Amperometric measurements of pyocyanin in chemical mixture (CM) background. (A) Current decay as function of time for pyocyanin concentrations of 1-90 $\mu \mathrm{M}$. (B) Current measurements for submicromolar scale. Inset shows the linearity of the accumulated charge obtained from each of the amperometric measurements. 


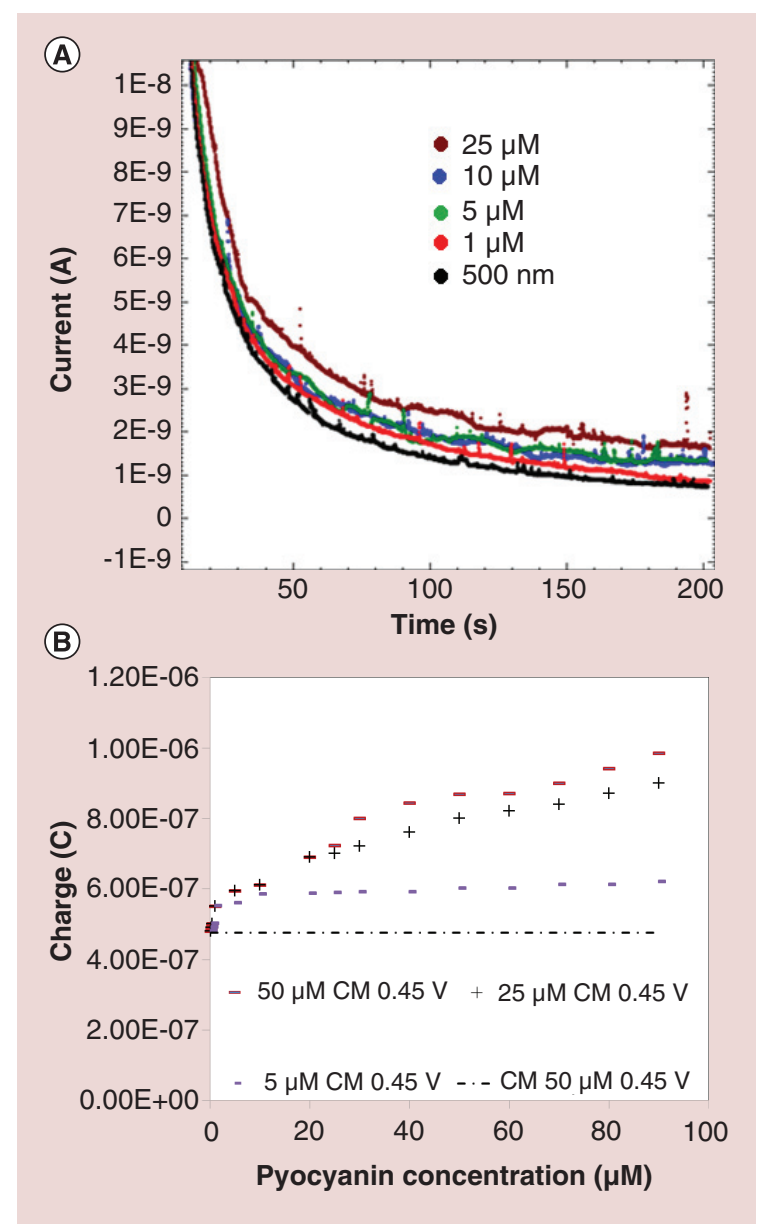

Figure 6. Pyocyanin detection in chemical mixture (CM) at $0.45 \mathrm{~V}$. (A) Amperometric measurements of pyocyanin in $50 \mu \mathrm{M} \mathrm{CM}$ at $0.45 \mathrm{~V}$. (B) The accumulated charge obtained from the amperometric measurements for pyocyanin detected in 5, 25 and $50 \mu \mathrm{M} \mathrm{CM}$, respectively. The background measurement of pure $50 \mu \mathrm{M}$ CM is shown as a baseline. The change in slope of the three datasets appears when the pyocyanin concentration is greater than the background CM concentration.

higher than the detection limit obtained by amperometry, demonstrating that the amperometric technique can provide lower detection limits of pyocyanin than spectrophotometry.

\section{Detection of bacterial production of pyocyanin in artificial sputum}

Pyocyanin produced by $P$. aeruginosa was detected directly in the culture using a constant potential of 0.82 $\mathrm{V}$. The measurements shows that an increasing accumulated charge can be obtained by increasing pyocyanin concentration (Figure 8). This indicates that the increase in signal is due to the pyocyanin in the samples.

The accumulated charge was compared with the calibration curve obtained in Figure 5 which indicates that around $14 \mu \mathrm{M}$ pyocyanin was detected in the investigated culture. Since the calibration curve is conducted in the CM medium, this pyocyanin concentration is only an approximation; however, it is a validation of the ability of detecting pyocyanin by amperometry at positive potentials.

\section{Discussion}

The common approach for diagnosing bacterial infections is by microbiological culturing in a predetermined culture media under controlled laboratory conditions. If growth appears, the quantification can be made. However, clinical diagnosis is not possible if the concentration of bacteria in patient samples is less than $10^{5}$ cells $/ \mathrm{ml}$. Therefore, pyocyanin as a biomarker for $P$. aeruginosa could be an alternative to direct bacterial detection.

Currently, laboratory-based methods are not suitable for ultrasensitive pyocyanin sensing. The detection is limited by interfering molecules in complex sample media such as human sputum and human saliva. Samples from patients would need extensive pretreatment and up-concentration of pyocyanin. The current measurement techniques such as spectrophotometry have shown that despite measuring pure samples, it was not possible to measure submicromolar concentrations.

Electrochemical detection of pyocyanin is a rapid and sensitive technique for detecting presence of $P$. aeruginosa. Amperometry is a potential-specific method that can be used for high sensitivity detection of pyocyanin in complex backgrounds. Amperometry has to the authors' knowledge never been used to detect pyocyanin using a standard single WE due to the difficulty in filtering signals from interfering compounds. Only one earlier study has investigated amperometry in pyocyanin detection in a relatively complicated setup with two WEs in a microfabricated nanofluidic assembly [23]. Instead, we have shown that simple commercial electrodes can be used if the constant potential is relatively high.

In this paper, we demonstrate the ability to detect and quantify pyocyanin in complex solutions using amperometry in a standard three-electrode configuration. Our method provides a promising approach for direct sensitive screening of biofluids for $P$. aeruginosa. This can significantly help in the early diagnosis of bacterial infections.

We have demonstrated the ability of the amperometric detection to sense pyocyanin over the range of $125 \mathrm{nM}-100 \mu \mathrm{M}$ in complex backgrounds. The ultrasensitivity in detection was achieved through defining the optimal potential at which pyocyanin can be quantified with constant background contribution. The point is that the background signal contribution can be constant despite the variance in the actual background concentration at this particular optimal potential. In 
addition, the integration of the current over a time of $200 \mathrm{~s}$ resulted in measures with reduced noise.

It is yet unknown how the pyocyanin concentration correlates to the bacterial concentration as it can differ from isolate to another. However, being able to detect nanomolar ranges of pyocyanin will make it possible to determine if any, usually undetectable, $P$. aeruginos $a$ is present in a sample.

This method can in fact be applied at any solution with unknown background concentration. The essence is to identify the best $\mathrm{S} / \mathrm{N}$ over a range of potentials and apply this potential for the quantification experiments using amperometry. This is especially relevant when detecting pyocyanin in patient samples such as sputum from patients with lung infections. The background interference in the samples might differ from patient to patient, thus this method is a straightforward way to selectively quantify pyocyanin. This can be extremely useful when measuring in sputum samples collected from children, since expectorates usually contain a significant but yet unknown amount of water and saliva.

The WE in this study consisted of screenprinted gold. It is believed that detection limits beyond the nanomolar range can be achieved if the electrode surface is enhanced by nanotechnology. It can be done by using nanoelectrodes or by simply modifying the electrodes with conductive nanoparticles that will increase the specific surface area and increase the sensitivity. Thus, this study has only demonstrated a proof-ofconcept for simple amperometric pyocyanin detection and there is yet room for optimization.

\section{Conclusion}

Detection of pyocyanin as a biomarker for $P$. aeruginosa was demonstrated with high reproducibility by amperometry. The determination of pyocyanin was conducted in the presence of redox-active compounds. The lowest detected pyocyanin value was in the submicromolar range while a higher detection limit was obtained by spectrophotometry. An important finding is that the contribution of the background mixture reached a saturation level at high concentrations, allowing unaffected detection of pyocyanin regardless of the concentration of the interfering background. Finally, this method has been validated by measuring the pyocyanin secretion in $P$. aerugonosa cultures in artificial sputum medium. This pyocyanin detection procedure has great potential of being a rapid point-ofcare diagnostic technique of $P$. aeruginosa infections as no sample pretreatment is needed.

Financial \& competing interests disclosure

HK Johansen was funded by a clinical research stipend from

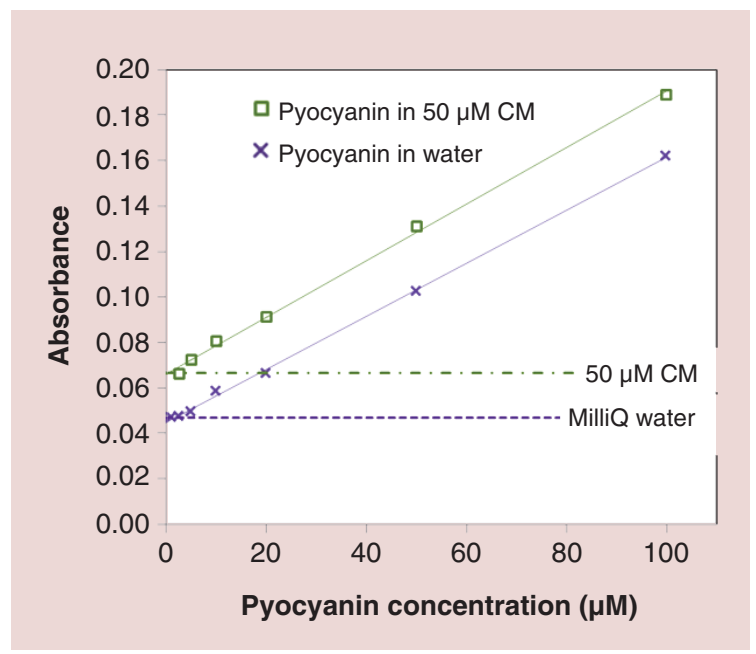

Figure 7. Spectrophotometric detection limit of pyocyanin. Pyocyanin in water has a detection limit of $1 \mu \mathrm{M}$. The pyocyanin detection limit in $50 \mu \mathrm{M}$ chemical mixture (CM) is $2.5 \mu \mathrm{M}$. The respective backgrounds of water and $50 \mu \mathrm{M}$ CM are plotted as dotted lines. The measurements were performed three-times for each point with identical resulting absorbance.

The Novo Nordisk Foundation and Rigshospitalet Rammebevilling 2015-17 and Lundbeckfonden Grant R167-201315229. The authors have no other relevant affiliations or financial involvement with any organization or entity with a financial interest in or financial conflict with the subject matter or materials discussed in the manuscript apart from those disclosed.

No writing assistance was utilized in the production of this manuscript.

\section{Open access}

This work is licensed under the Creative Commons Attribution-NonCommercial 4.0 Unported License. To view a copy of this license, visit http://creativecommons.org/licenses/bync-nd/4.0/

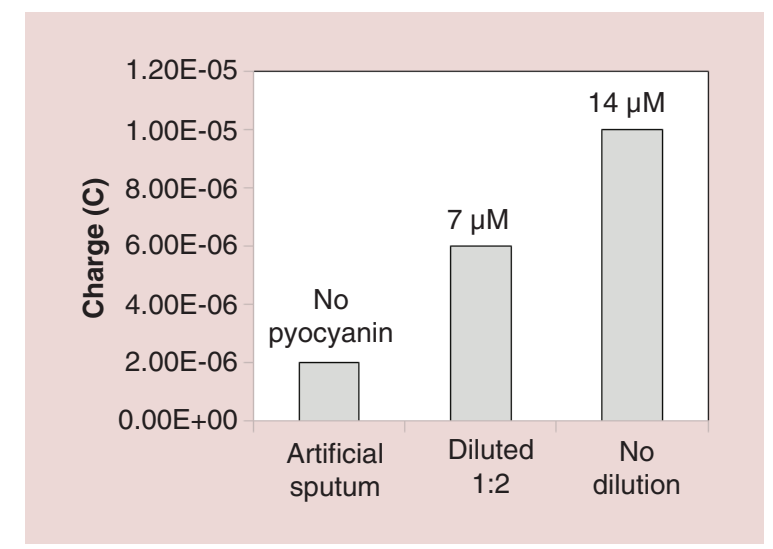

Figure 8. Amperometric detection of pyocyanin in bacteria cultured in artificial sputum medium. The accumulated charge shows increasing signal with increasing concentration. 
Executive summary

Pyocyanin as a biomarker for Pseudomonas aeruginosa

- Pseudumonas aeruginosa is a multidrug-resistant bacterium responsible for high morbidity and mortality rates in humans.

- Pyocyanin is an electroactive molecule uniquely produced by $P$. aeruginosa which makes it an exclusive biomarker for this bacterium.

Amperometric biosensing

- The amperometric biosensing technique involves applying a constant electric potential to a sample and measuring the output current.

- Pyocyanin has never been tested by amperometry using a three-electrode configuration due to the complexity in isolating the target signal.

Selective detection signal

- The commonly used pyocyanin peak overlaps with the peaks of artificial sputum and human saliva which makes it not useful in amperometric measurements of body fluids of patients.

- A relatively high characteristic peak value for pyocyanin has been demonstrated to be outside background overlap. The electrochemical precharacterization provides a unique technique to detect pyocyanin without the need for a label or external charged molecule.

Detection of pyocyanin in complex background

- A detection limit of $125 \mathrm{nM}$ with a $\mathrm{r}^{2}=0.999(n=5)$ with a measurement time of $200 \mathrm{~s}$ per sample.

- The method was validated in a bacterial culture in artificial sputum medium.

\section{References}

1 Miller LC, O’Loughlin CT, Zhang Z et al. Development of potent inhibitors of pyocyanin production in Pseudomonas aeruginosa. J. Med. Chem. 58, 1298-1306 (2015).

2 Damkiær S, Yang L, Molin S, Jelsbak L. Evolutionary remodeling of global regulatory networks during long-term bacterial adaptation to human hosts. Proc. Natl Acad. Sci. USA 110(19), 7766-7771 (2013).

3 Abu EA, Su S, Sallans L et al. Cyclic voltammetric, fluorescence and biological analysis of purified aeruginosin A, a secreted red pigment of Pseudomonas aeruginosa PAO1. Microbiology 159, 1736-1747 (2013).

4 Pruett T. Bad bugs, no drugs: no ESKAPE! An update from the Infectious Diseases Society of America. Clin Infect Dis. 48(1), 1-12 (2009).

5 Wu H, Moser C, Wang HZ, Hoiby N, Song ZJ. Strategies for combating bacterial biofilm infections. Int. J. Oral Sci 7(1), 1-7 (2015).

6 Hung CS, Henderson JP. Emerging concepts of biofilms in infectious diseases. Mol. Med. 106(4), 292-296 (2009).

7 Llor C, Bjerrum L. Antimicrobial resistance: risk associated with antibiotic overuse and initiatives to reduce the problem. Ther. Adv. Drug Saf. 5(6), 229-241 (2014).

8 Bren L. Battle of the bugs: fighting antibiotic resistance. FDA Consum. 36(4), 28-34 (2002).

9 O'Neill J. Rapid diagnostics: stopping unnecessary use of antibiotics. In: Review on Antimicrobial Resistance (2015).

10 Michel-Briand Y, Baysse C. The pyocins of Pseudomonas aeruginosa. Biochimie 84, 499-510 (2002).

11 Jayaseelan S, Ramaswamy D, Dharmaraj S. Pyocyanin: production, applications, challenges and new insights. World J Microb. Biotechnol. 30, 1159-1168 (2014).

12 Dietrich LE, Price-Whelan A, Petersen A, Whiteley M, Newman DK. The phenazine pyocyanin is a terminal signalling factor in the quorum sensing network of Pseudomonas aeruginosa. Mol. Microbiol. 61, 1308-1321 (2006).

13 Alatraktchi FA, Breum Andersen S, Johansen HK, Molin S, Svendsen WE. Fast selective detection of pyocyanin using cyclic voltammetry. Sensors 16,3 (2016).

14 Alatraktchi FA, Bakmand T, Dimaki M, Svendsen WE. Novel membrane-based electrochemical sensor for real-time bio-applications. Sensors 14, 22128-22139 (2014).

15 Svendsen WE, Alatraktchi FA, Bakmand T, Waagepetersen $\mathrm{H}$, Dimaki M. Novel culturing platform for brain slices and neuronal cells. IEEE Eng. Med. Biol. Soc. 3299, 346-349 (2015).

16 Seviour T, Doyle LE, Lauw SJ et al. Voltammetric profiling of redox-active metabolites expressed by Pseudomonas aeruginosa for diagnostic purposes. Chem. Commun. 51, 3789-3792 (2015).

17 Sismaet HJ, Webster TA, Goluch ED. Up-regulating pyocyanin production by amino acid addition for early electrochemical identification of Pseudomonas aeruginosa. Analyst 139, 4241-4246 (2014).

18 Sharp D, Gladstone P, Smith RB, Forsythe S, Davis J. Approaching intelligent infection diagnostics: carbon fibre sensor for electrochemical pyocyanin detection. Bioelectrochemistry 77, 114-119 (2009).

19 Webster TA, Sismaet HJ, Conte JL, Chan IC, Goluch ED. Electrochemical detection of Pseudomonas aeruginosa in human fluid samples via pyocyanin. Biosens. Bioelectron. 60, 265-270 (2014).

20 Price-Whelan A, Dietrich LE, Newman DK. Pyocyanin alters redox homeostasis and carbon flux through central metabolic pathways in Pseudomonas aeruginosa PA14. J. Bacteriol. 189(17), 6372-6381 (2007). 
21 Landers B. Oral bacteria: how many? How fast? www.rdhmag.com

22 Kirchner S, Fothergill JL, Wright EA, James CE, Mowat E, Winstanley C. Use of artificial sputum medium to test antibiotic efficacy against Pseudomonas aeruginosa in conditions more relevant to the cystic fibrosis lung. J. Vis. Exp. 64 (2012).

23 Webster TA, Sismaet HJ, Goluch ED. Amperometric detection of pyocyanin in nanofluidic channels. Nano LIFE 3 (2013). 